\title{
Steady-state crack growth and fracture work based on the theory of mechanism-based strain gradient plasticity
}

\author{
Y. Wei ${ }^{\mathrm{a}, *}$, X. Qiu ${ }^{\mathrm{b}}$, K.C. Hwang ${ }^{\mathrm{b}}$ \\ ${ }^{a}$ LNM, Institute of Mechanics, Chinese Academy of Sciences, Beijing 100080, PR China \\ ${ }^{\mathrm{b}}$ Failure Mechanics Laboratory, Department of Engineering Mechanics, Tsinghua University, Beijing 100084, PR China
}

Received 17 October 2001; received in revised form 12 December 2002; accepted 23 December 2002

\begin{abstract}
Mode I steady-state crack growth is analyzed under plane strain conditions in small scale yielding. The elastic-plastic solid is characterized by the mechanism-based strain gradient (MSG) plasticity theory [J. Mech. Phys. Solids 47 (1999) 1239, J. Mech. Phys. Solids 48 (2000) 99]. The distributions of the normal separation stress and the effective stress along the plane ahead of the crack tip are computed using a special finite element method based on the steady-state fundamental relations and the MSG flow theory. The results show that during the steady-state crack growth, the normal separation stress on the plane ahead of the crack tip can achieve considerably high value within the MSG strain gradient sensitive zone. The results also show that the crack tip fields are insensitive to the cell size parameter in the MSG theory. Moreover, in the present research, the steady-state fracture toughness is computed by adopting the embedded process zone (EPZ) model. The results display that the steady-state fracture toughness strongly depends on the separation strength parameter of the EPZ model and the length scale parameter in the MSG theory. Furthermore, in order for the results of steady crack growth to be comparable, an approximate relation between the length scale parameters in the MSG theory and in the Fleck-Hutchinson strain gradient plasticity theory is obtained.
\end{abstract}

(c) 2003 Elsevier Ltd. All rights reserved.

Keywords: Steady-state crack growth; Crack tip fields; Fracture toughness; Mechanism-based strain gradient plasticity

\section{Introduction}

The material fracture behavior is frequently assessed by a criterion parameter, the fracture toughness, which characterizes the work of crack initiation or steady-state crack growth. The crack initiation work corresponds to the initial fracture toughness $\left(\Gamma_{\text {in }}\right)$, while the steady-state growth work corresponds to the steady-state fracture toughness $\left(\Gamma_{\mathrm{ss}}\right)$, as sketched in Fig. 1(a). Both the initiation criterion and the steadystate growth criterion are frequently applied to the elastic material and the elastic-plastic material, respectively. For the elastic homogeneous material, the initial fracture toughness and the steady-state fracture toughness are the same values [1]. However, for the elastic-plastic material, they are different, as shown in

\footnotetext{
${ }^{*}$ Corresponding author. Tel.: +86-10-62648721; fax: +86-10-62561284.

E-mail address: ywei@lnm.imech.ac.cn (Y. Wei).
} 


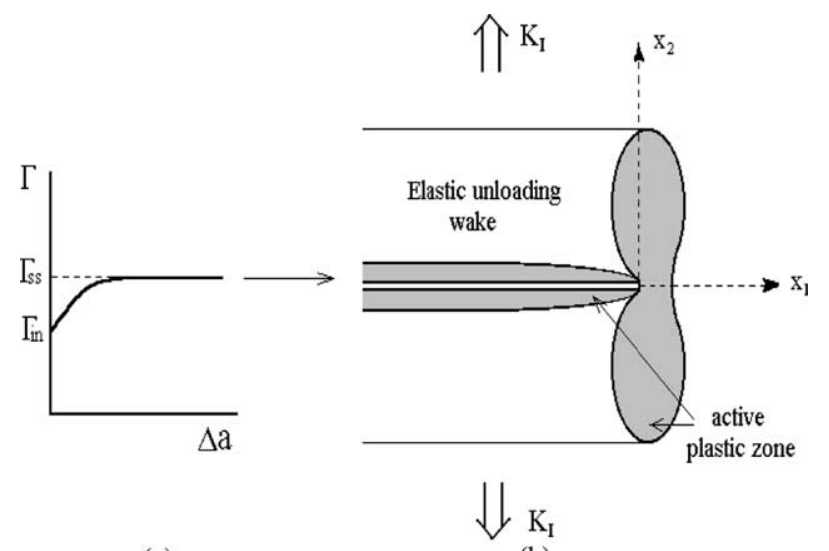

(a)

(b)

Fig. 1. Sketch of the steady-state crack growth is shown. (a) Fracture toughness curve and steady-state fracture toughness; (b) active plastic zone for a mode I steady-state crack.

Fig. 1(a). Before the steady-state crack growth is achieved, the crack has undergone a long stable advance after initiation. Since the steady-state crack growth is the critical fracture state for the elastic-plastic material, the measurements of the steady-state fracture toughness and the related researches have attracted a great deal of interest [2-12].

About the steady-state crack growth study using the conventional elastic-plastic theory, many researches have been published in the past decades. Only a part of those are mentioned here. Dean and Hutchinson [13] and Parks et al. [14] investigated the elastic-plastic crack tip fields for the steady-state crack growth under small scale yielding condition. These authors developed a special finite element method based on the steady-state conditions by transferring the flow theory equations from time-rate form into space-rate form. They used the special finite element method to calculate the steady-state crack tip fields successfully. Gao and Hwang [15], Drugen et al. [16] and later Hwang and Luo [17] studied the steady-state crack tip fields. Using the finite element method presented by Dean and Hutchinson [13], Varias and Shih [18] computed the steady-state crack tip field for the model I crack when T-stress effect was considered; Suo et al. [19] and Beltz et al. [6] investigated the steady-state fracture toughness by adopting a plasticity-free strip model (SSV model). Tvergaard and Hutchinson [2,3] studied the fracture work for the mode I homogeneous material and bi-material using the embedded process zone (EPZ) model. Wei and Hutchinson [7] used the SSV model and the EPZ model to study the steady-state fracture work for the thin film delamination and the thin film peeling test problem [8]. The same authors [9] investigated the model sensitivities by presenting and adopting a unified model.

Note that from previous research for the steady-state crack growth using the conventional elastic-plastic theory, the predicted peak separation stresses ahead of the crack tip are no larger than about 4-6 times the yielding stress, no matter what separation models are used. However, there have been more and more experimental evidences indicating that the higher separation stresses likely exist near the growing crack tip $[4,5,10,20]$. Moreover, micro-scale predictions from the first principle calculations have shown that for the metal/ceramic interface, the separation strength can achieve 30 or even 40 times the yielding stress of the metal [21,22]. Obviously, the previous research has implied that the conventional elastic-plastic theory fails to account for the separation stress elevation. The differences between the fracture behaviors at the macroscale fracture and at the meso-scale fracture are referred to as the size effects in fracture mechanics. Simultaneously, the size effects have been found in the other problems, such as the micro-indentation tests [23-26], the torsion test for thin copper wires [27], as well as the bending test of thin beams [28]. In order to 
simulate the size effects, several versions of the strain gradient plasticity theories have recently been developed [29-34]. Using the strain gradient plasticity theories, the size effects can be characterized and simulated. Some applications of the strain gradient plasticity theories to the simulations of the size effects have been presented, such as the simulations for the micro-indentation tests [26,35-37] and for the stationary and growing crack tip fields, as well as for the thin film delamination [38-43]. Particularly, Wei and Hutchinson [39] using the Fleck-Hutchinson's strain gradient plasticity theory have studied the steady-state crack growth problem and attained that the maximum separation stress near the crack tip can achieve 10 times the material yielding stress. This result has promoted the considerable interest for further efforts to explore the linkage of the macroscopic fracture with the atomistic fracture [20].

In the present study, the steady-state crack growth is analyzed for a sharp macroscopic crack in an elastic-plastic material, which is characterized by the MSG strain gradient plasticity theory [33,34]. Like other strain gradient theories, the MSG theory includes a length scale parameter. Suppose that the mode I crack extends over a sufficient distance, so that an observer traveling with the crack tip sees no variation in the mechanical fields, as shown in Fig. 1(b). For comparing with the Fleck-Hutchinson strain gradient plasticity theory, the MSG theory will be assessed in detail. The crack tip fields and the steady-state fracture work will be figured out. Additionally, the cell size effects in the MSG theory, and the connections between the MSG theory and the Fleck-Hutchinson strain gradient plasticity theory will be explored. The crack tip fields, i.e., the distributions of the normal separation stress and the effective stress on the plane ahead of the growing crack tip will be analyzed using the special finite element method based on the steady-state conditions and the MSG theory. The steady-state fracture work or fracture toughness will be analyzed by adopting the EPZ model. In order for the results of steady crack growth to be comparable, an approximate relation between the length scale parameters in the MSG theory and in the Fleck-Hutchinson strain gradient plasticity theory will be presented.

\section{The MSG flow theory}

The MSG flow theory has been derived by Qiu et al. [44] following the derivation line of the MSG deformational theory $[33,34]$. The procedures can be summarized as follows: (1) Consider that a micro-scale cell, with the cell size $l_{\varepsilon}$ and with the geometrically necessary dislocation density $\rho_{\mathrm{G}}$, is homogenized to an equivalent solid characterized by the elastic-plastic theory plus a hardening law based on the Taylor model [45]. (2) Applying the conventional volume average scheme to the micro-scale cell, one can derive the rateindependent relations for the MSG flow theory. The results are listed as follows:

$$
\begin{gathered}
\dot{\sigma}_{i j}=K \dot{\varepsilon}_{k k} \delta_{i j}+2 \mu\left(\dot{\varepsilon}_{i j}^{\prime}-\alpha^{\prime} \frac{3 \sigma_{i j}^{\prime}}{2 \sigma} \dot{\varepsilon}^{\mathrm{p}}\right) \\
\dot{\tau}_{i j k}=c\left[\dot{\eta}_{i j k}+\frac{1}{2}\left(\dot{\eta}_{k i j}+\dot{\eta}_{k j i}\right)+\left(\frac{2 K}{\mu}-\frac{4}{3}\right) \dot{\eta}_{i j k}^{H}\right]-\alpha^{\prime}\left\{\frac{c}{1+a} \frac{3 \sigma_{m n}^{\prime}}{2 \sigma^{2}}\left(\sigma_{k i}^{\prime} \dot{\eta}_{j m n}+\sigma_{k j}^{\prime} \dot{\eta}_{i m n}\right)\right. \\
\left.\quad+\frac{1}{1+a} \frac{3}{4 \sigma^{2}} G_{i j k m n} \dot{\sigma}_{m n}^{\prime}-\left(\frac{e}{1+a}+\frac{3 \mu}{\sigma}\right) \frac{3}{4 \sigma^{2}} G_{i j k m n} \sigma_{m n}^{\prime} \dot{\varepsilon}^{\mathrm{p}}+\frac{3 \mu}{\sigma}\left(\tau_{i j k}-\frac{K l_{\varepsilon}^{2}}{6} \eta_{i j k}^{H}\right) \dot{\varepsilon}^{\mathrm{p}}\right\} \\
\dot{\varepsilon}^{\mathrm{p}}=\frac{1}{1+a} \frac{1}{\sigma}\left(\sigma_{i j}^{\prime} \dot{\varepsilon}_{i j}-\alpha^{2} \mu b \frac{3}{4 \eta} \eta_{i j k}^{\prime} \dot{\eta}_{i j k}\right)
\end{gathered}
$$

where the flow criterion is expressed in terms of the Mises' effective stress [33,34,37] 


$$
\sigma_{\mathrm{e}}=\sigma=\sqrt{\sigma_{0}^{2} f_{\mathrm{p}}^{2}\left(\varepsilon^{\mathrm{p}}\right)+18 \alpha^{2} \mu^{2} b \eta}=\sigma_{0} \sqrt{f_{\mathrm{p}}^{2}\left(\varepsilon^{\mathrm{p}}\right)+l \eta}
$$

and

$$
\alpha^{\prime}=0 \quad \text { if } \sigma_{\mathrm{e}}<\sigma, \text { or } \sigma_{\mathrm{e}}=\sigma \text { and } \dot{\varepsilon}^{\mathrm{p}}<0 ; \quad \alpha^{\prime}=1 \quad \text { if } \sigma_{\mathrm{e}}=\sigma \text { and } \dot{\varepsilon}^{\mathrm{p}} \geqslant 0
$$

The parameters and variables in the above relations are defined as

$$
\begin{aligned}
& c=\frac{\mu l_{\varepsilon}^{2}}{12}, \quad a=\frac{\sigma_{0}^{2}}{3 \mu \sigma} f_{\mathrm{p}} f_{\mathrm{p}}^{\prime}, \quad e=\frac{f_{\mathrm{p}}^{\prime}}{f_{\mathrm{p}}}+\frac{f_{\mathrm{p}}^{\prime \prime}}{f_{\mathrm{p}}^{\prime}} \\
& G_{i j k m n}=\sigma_{k i}^{\prime}\left(\tau_{j m n}+\tau_{j n m}-\tau_{m n j}\right)+\sigma_{k j}^{\prime}\left(\tau_{i m n}+\tau_{i n m}-\tau_{m n i}\right)
\end{aligned}
$$

where $f_{\mathrm{p}}\left(\varepsilon^{\mathrm{p}}\right)$ is the function in the uni-axial stress-plastic strain relation determined from uni-axial tension:

$$
\sigma_{\mathrm{I}}=\sigma_{0} f_{\mathrm{p}}\left(\varepsilon^{\mathrm{p}}\right)
$$

$\sigma_{0}$ is a reference stress; $\varepsilon^{\mathrm{p}}$ is accumulative plastic strain; $b$ is the Burgers vector; $\alpha$ is an empirical coefficient in the Taylor model and is taken the value around 0.3 for the conventional metals; $K=E / 3(1-2 v)$ and $\mu=E / 2(1+v)$ are the elastic bulk modulus and shear modulus, respectively; $l_{\varepsilon}$ is the cell size, and from analysis of Gao et al. [33], Huang et al. [37] and Qiu et al. [44],

$$
l_{\varepsilon}=10 \mu b / \sigma_{\mathrm{Y}}, \quad l=18 \alpha^{2}\left(\frac{\mu}{\sigma_{0}}\right)^{2} b
$$

$l$ is the length scale parameter characterizing the strain gradient strength $[33,37] ; \eta$ is the effective strain gradient and can be expressed by the components of strain gradient $\eta_{i j k}=u_{k, i j}$ as

$$
\eta=\sqrt{\frac{1}{4} \eta_{i j k}^{\prime} \eta_{i j k}^{\prime}}
$$

and the deviatoric and volumetric components of $\eta_{i j k}$ are dictated as [30]

$$
\eta_{i j k}^{\prime}=\eta_{i j k}-\eta_{i j k}^{H}, \quad \eta_{i j k}^{H}=\frac{1}{4}\left(\delta_{i k} \eta_{j \mathrm{pp}}+\delta_{j k} \eta_{i \mathrm{pp}}\right)
$$

A piecewise power law hardening stress-strain relation is considered to characterize the solid behaving at macro-scale,

$$
\sigma_{\mathrm{I}}=E \varepsilon, \quad \text { for } \varepsilon \leqslant \frac{\sigma_{\mathrm{Y}}}{E} ; \text { and } \quad \sigma_{\mathrm{I}}=\sigma_{0} \varepsilon^{N}, \quad \text { for } \varepsilon \geqslant \frac{\sigma_{\mathrm{Y}}}{E}
$$

where $N$ is material strain hardening exponent. From (7) and (11), one has

$$
\sigma_{0}=\sigma_{\mathrm{Y}}\left(E / \sigma_{\mathrm{Y}}\right)^{N}
$$

Through checking the relations from (1) to (10), and comparing the relations of the MSG flow theory with the conventional elastic-plastic flow theory relations, obviously, the additional terms, effective strain gradient and its rate, are included in the MSG flow theory. The strain gradient strength is characterized by the length parameter $l$. When the length parameter is taken as zero, the MSG flow theory will degenerate to the conventional elastic-plastic flow theory. According to the research for the MSG theory in Gao et al. [33], the value of the length parameter $l$ falls within the region of $1-10 \mu \mathrm{m}$ for the typical metal materials.

\section{Numerical formulation for steady-state mode I crack growth}

A semi-infinite crack, depicted in Fig. 1, steadily advancing within the solid characterized by the MSG flow theory, will be analyzed. For the mode I crack tip stress field, the strain gradient effects are weak and 
can be neglected at the points far away from the crack tip. When strain gradient effects are small, the stress field can be described approximately by the classical elastic mode I solution with a $r^{-1 / 2}$ dependence and with the intensity factor $K_{\mathrm{I}}$. Within the active plastic zone, there exists a strain gradient dominated zone surrounding the crack tip. The numerical method for the steady-state crack propagation will be discussed in this section.

\subsection{Steady-state formulation of the MSG flow theory}

The rate-independent constitutive relations of the MSG flow theory (formula (1)-(3)) can be expressed in a more compact form, as shown in Appendix A. For the steady-state crack growth and when crack advances along the $x_{1}$ direction (Fig. 1), the rate quantities can be expressed as

$$
\left(\dot{\tau}_{i j k}, \dot{\eta}_{i j k}, \dot{\sigma}_{i j}, \dot{\varepsilon}_{i j}\right)=-\dot{a}\left(\frac{\partial \tau_{i j k}}{\partial x_{1}}, \frac{\partial \eta_{i j k}}{\partial x_{1}}, \frac{\partial \sigma_{i j}}{\partial x_{1}}, \frac{\partial \varepsilon_{i j}}{\partial x_{1}}\right)
$$

where $\dot{a}$ is the crack tip advance velocity. Substituting (13) into (A.1) in Appendix A, the equations, independent of the crack tip velocity, are obtained in matrix form as

$$
\left\{\begin{array}{l}
\frac{\partial \boldsymbol{\sigma}}{\partial x_{1}} \\
\frac{\partial \tau}{\partial x_{1}}
\end{array}\right\}=\left[\begin{array}{cc}
\mathbf{A} & \mathbf{B} \\
\mathbf{A}^{\prime} & \mathbf{B}^{\prime}
\end{array}\right]\left\{\begin{array}{l}
\frac{\partial \boldsymbol{\varepsilon}}{\partial x_{1}} \\
\frac{\partial \boldsymbol{\eta}}{\partial x_{1}}
\end{array}\right\}
$$

where $\boldsymbol{\sigma}, \boldsymbol{\varepsilon}, \boldsymbol{\tau}$ and $\boldsymbol{\eta}$ are the matrices of stress, strain, higher-stress and higher-strain, the tensor expressions of $\mathbf{A}, \mathbf{B}, \mathbf{A}^{\prime}$ and $\mathbf{B}^{\prime}$ are shown in (A.2)-(A.5) of Appendix A.

From Eq. (14), the rate-form constitutive relation of flow theory has been transferred into a special constitutive equation in full quantities, additionally, with differentiation with respect to the coordinate $x_{1}$. Thus, one only needs to solve such a problem as (14), similar to a deformational plastic problem using the iteration method [13,14], rather than using the usual scheme for solving the elastic-plastic flow theory problem with loading step-by-step.

\subsection{Variational equation for the steady-state crack growth}

Based on Eq. (14) and the discussions in the last subsection, one may directly obtain the full quantity stress and strain fields for the steady-state crack growth problem. Variational equation with full quantity variables for strain gradient solid can be dictated as [30]

$$
\int_{V}\left(\sigma_{i j} \delta \varepsilon_{i j}+\tau_{i j k} \delta \eta_{i j k}\right) \mathrm{d} V=\int_{V} f_{k} \delta u_{k} \mathrm{~d} V+\int_{S} t_{k} \delta u_{k} \mathrm{~d} S+\int_{S} r_{k}\left(D \delta u_{k}\right) \mathrm{d} S
$$

where the traction and torque on a surface $S$ are defined by

$$
t_{k}=n_{i}\left(\sigma_{i k}-\frac{\partial \tau_{i j k}}{\partial x_{j}}\right)+n_{i} n_{j} \tau_{i j k}\left(D_{\mathrm{p}} n_{\mathrm{p}}\right)-D_{j}\left(n_{i} \tau_{i j k}\right), \quad r_{k}=n_{i} n_{j} \tau_{i j k}
$$

Here $t_{k}, r_{k}$ and $n_{i}$ are the traction, torque and the external normal direction cosine, respectively, and $f_{k}$ is body force. The differential operators $D$ and $D_{j}$ in (15) and (16) are defined as [30]

$$
D_{j}=\partial() / \partial x_{j}-n_{j} n_{k} \partial() / \partial x_{k}, \quad D=n_{k} \partial() / \partial x_{k}
$$

Based on Eq. (15), the finite element formulation for strain gradient solid can be developed (see Section 3.3). 


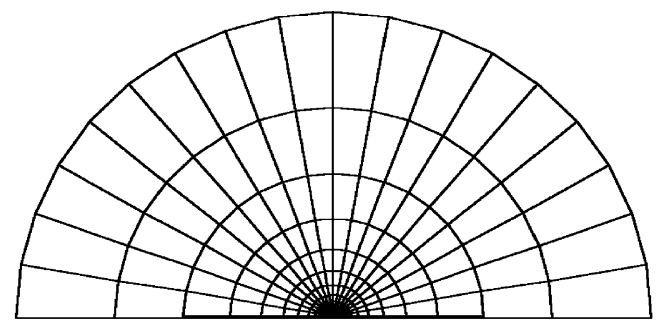

(a)

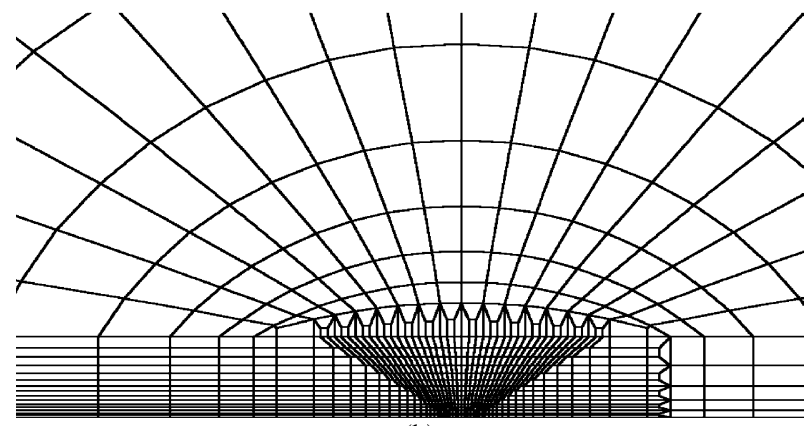

(b)

Fig. 2. Finite element mesh. (a) Global mesh, and (b) near tip mesh.

Based on the variational equation (15), let us discuss the boundary conditions for the plane strain mode I crack growth problem. Consider no body force case in the present research, $f_{k}=0$. Referring to Fig. 1, considering symmetry about the line ahead of the crack tip, one only needs to consider the upper-half plane $\left(x_{2} \geqslant 0\right)$. Referring to Figs. 1 and 2, boundary conditions can be described as

$$
\left\{\begin{array}{l}
\text { Conventional } K_{\mathrm{I}} \text {-field, for } x_{1}^{2}+x_{2}^{2} \rightarrow \infty \\
t_{1}=0, t_{2}=0, r_{1}=0, r_{2}=0, \quad \text { for } x_{1}<0, x_{2}=0 \\
u_{2}=0, \frac{\partial u_{1}}{\partial x_{2}}=0, t_{1}=0, r_{2}=0, \quad \text { for } x_{1}>0, x_{2}=0
\end{array}\right.
$$

From the first line of Eq. (18), since strain gradient effect is very weak and can be neglected at the remote boundary, boundary conditions corresponding to conventional $K_{\mathrm{I}}$-field are exerted. On the crack surface, tractions are prescribed. On the surface of symmetry ahead of the crack tip, boundary condition is a mixed one, as shown in the third line of Eq. (18), where torque condition $r_{2}=0$ comes from the condition $r_{2}=\tau_{222}$ from (16) and $\tau_{222}=0$ due to symmetry.

\subsection{Finite element method for the steady-state problem}

The special finite element method [13,14], in which the iteration procedures to solve the special stressstrain differential equation (see (14)) are employed, is used to obtain the convergent solution directly. The finite element approach and the solution procedures are outlined below.

Anticipating a numerical implementation within a finite element framework, let $\mathbf{E}$ be a generalized strain vector with components comprised of both the strains and the strain gradients, and let $\boldsymbol{\Sigma}$ be the stress vector 
containing components of stress and higher-stress. The matrix of incremental moduli for plastic loading is denoted by $\mathbf{D}$ such that $\dot{\boldsymbol{\Sigma}}=\mathbf{D} \dot{\mathbf{E}}$, and the expression of $\mathbf{D}$ can be found in (A.1) of Appendix A or in (14). Let $\mathbf{U}$ be the vector of nodal displacements and let $\mathbf{B}$ be the strain matrix such that $\mathbf{E}=\mathbf{B U}$. The finite element problem for $\mathbf{U}$ in terms of applied boundary nodal forces $\mathbf{F}$ and any specified generalized plastic strain $\mathbf{E}^{\mathrm{p}}$ is represented in the standard matrix notation as (see Eq. (15))

$$
\mathbf{K}^{\mathrm{e}} \mathbf{U}=\int_{S} \mathbf{N}^{\mathrm{T}} \mathbf{F} \mathrm{d} S+\int_{V} \mathbf{B}^{\mathrm{T}} \mathbf{D}^{\mathrm{e}} \mathbf{E}^{\mathrm{P}} \mathrm{d} V \quad \text { where } \quad \mathbf{K}^{\mathrm{e}}=\int_{V} \mathbf{B}^{\mathrm{T}} \mathbf{D}^{\mathrm{e}} \mathbf{B} \mathrm{d} V
$$

where the superscripts "e" and "p" represent the elastic part and plastic part for corresponding quantities. Contribution of the applied boundary nodal forces $\mathbf{F}$ on the equilibrium relation (19) can be figured out from three terms at the right-hand side of variational equation (15).

The iteration steps are as follows:

1. Use the distribution of $\mathbf{E}^{\mathrm{p}}$ from the previous iteration in (19) to determine $\mathbf{U}$. In the first iteration take $\mathbf{E}^{\mathrm{p}}=\mathbf{0}$.

2. Compute $\mathbf{E}$ from $\mathbf{U}$.

3. Obtain a new estimate of the distribution of $\boldsymbol{\Sigma}$. Use $\boldsymbol{\Sigma}=\mathbf{D}^{\mathrm{e}} \mathbf{E}$ in the region upstream of the current estimate of active plastic zone and use $\mathbf{\Sigma}=\mathbf{D}^{\mathrm{e}}\left(\mathbf{E}-\mathbf{E}^{\mathrm{p}}\right)$ downstream from the active plastic zone. Where yield is currently met, make use of the fact that for steady-state growth, $\dot{\boldsymbol{\Sigma}}=\mathbf{D} \dot{\mathbf{E}}$ can be replaced by $\partial \mathbf{\Sigma} / \partial x_{1}=\mathbf{D} \partial \mathbf{E} / \partial x_{1}$ such that for any point $\left(x_{1}, x_{2}\right)$ within the active plastic zone

$$
\boldsymbol{\Sigma}\left(x_{1}, x_{2}\right)=\boldsymbol{\Sigma}\left(x_{1}^{*}, x_{2}\right)-\int_{x_{1}}^{x_{1}^{*}} \mathbf{D} \partial \mathbf{E} / \partial x_{1} \mathrm{~d} x_{1}
$$

where $\left(x_{1}^{*}, x_{2}\right)$ is corresponding point on the leading edge of the active plastic zone (i.e., the right edge of the active zone). The integration in (20) is performed for fixed $x_{2}$ and applies to all points within the active plastic zone.

4. Use $\mathbf{E}^{\mathrm{p}}=\mathbf{E}-\mathbf{D}^{\mathrm{e}^{-1}} \boldsymbol{\Sigma}$ to compute the new estimate of $\mathbf{E}^{\mathrm{p}}$ for the next iteration. Revise the active plastic zone using the new estimate of $\boldsymbol{\Sigma}$. To the left of the active plastic zone in the downstream unloading region, $\mathbf{E}^{\mathrm{p}}$ is a function only of $x_{2}$, corresponding to its value at the right edge of the active zone.

5. If satisfactory convergence has not been achieved, repeat steps $1-4$.

A finite element procedure with the equal-height mesh specially designed for the regions around the crack surface near crack tip to cope with the steady-state wake has been used to carry out the calculations, as discussed above and described in Dean and Hutchinson [13]. In the present research, the displacement of $K_{\mathrm{I}}$-field is exerted at the remote boundary. The total boundary conditions of the present mode I crack problem consist of the displacement boundary condition and the zero-traction boundary condition. Thus, the applied boundary nodal forces in Eq. (19) are equal to zero, $\mathbf{F}=\mathbf{0}$, from the right-hand side of Eq. (15). In the present analysis, the adopted finite element mesh for mode I steadily growing crack is shown in Fig. 2. In the calculation, the nine nodal iso-parametric displacement element (higher-order displacement element) will be adopted and the 2X2 Gauss integration points are selected for each element [39]. Under the pure displacement variables, the displacement condition $u_{2}=0$ on the surface of symmetry in (18) will be met on the nodes by exerting the constraints. The condition of symmetry $\partial u_{1} / \partial x_{2}=0$ will be satisfied automatically due to the prescription of the other conditions of symmetry $\left(u_{2}=0, t_{1}=0, r_{2}=0\right)$ exerted on the surface. The finite element methods for strain gradient solid have been assessed in Wei [43] and Chen et al. [46]. 


\section{Crack tip field for steady-state crack growth}

\subsection{Solution expressions}

After the steady-state crack growth problem is solved, one can compute the traction distribution along a plane with normal direction cosine $n_{i}$ by using formula (16). For the present mode I crack growth problem, and for the usually concerned plane ahead of the crack tip, along which the traction distributions are calculated, take $n_{1}=0$ and $n_{2}=1$ along $x_{1}>0$ and $x_{2}=0$. A remarkable feature of the strain gradient effect is that the stress distribution along the plane ahead of the crack tip is significantly increased as compared to conventional elastic-plastic theory results. Therefore the strain gradient plasticity theory is expected to explore the size effects for solids at the meso-scale or micro-scale, and the related research has attracted a great deal of interest recently.

From (16), one can calculate the normalized normal traction on the plane ahead of the crack tip

$$
\frac{t_{2}}{\sigma_{\mathrm{Y}}}=\left(\sigma_{22}-2 \frac{\partial \tau_{212}}{\partial x_{1}}-\frac{\partial \tau_{222}}{\partial x_{2}}\right) / \sigma_{\mathrm{Y}}=f\left(\frac{x_{1}}{l} ; \frac{E}{\sigma_{\mathrm{Y}}}, v, N, \frac{l_{\varepsilon}}{l}, \frac{K_{\mathrm{I}}}{\sigma_{\mathrm{Y}} l^{1 / 2}}\right)
$$

The effective stress is also a quantity of concern

$$
\frac{\sigma_{\mathrm{e}}}{\sigma_{\mathrm{Y}}}=g\left(\frac{x_{1}}{l} ; \frac{E}{\sigma_{\mathrm{Y}}}, v, N, \frac{l_{\varepsilon}}{l}, \frac{K_{\mathrm{I}}}{\sigma_{\mathrm{Y}} l^{1 / 2}}\right)
$$

Furthermore, in order to investigate the crack tip fields predicted using the strain gradient theory, the effective plastic strain along the plane ahead of crack tip will be calculated. From (4), (7) and (11), the effective plastic strain $\varepsilon^{\mathrm{p}}$ can be formulated in terms of the effective stress $\left(\sigma_{\mathrm{e}}\right)$ and the effective strain gradient $(\eta)$ as

$$
E \varepsilon^{\mathrm{p}} / \sigma_{\mathrm{Y}}=\left[\left(\frac{\sigma_{\mathrm{e}}}{\sigma_{\mathrm{Y}}}\right)^{2}-\left(\frac{E}{\sigma_{\mathrm{Y}}}\right)^{2 N} \operatorname{l\eta }\right]^{1 /(2 N)}-\left[\left(\frac{\sigma_{\mathrm{e}}}{\sigma_{\mathrm{Y}}}\right)^{2}-\left(\frac{E}{\sigma_{\mathrm{Y}}}\right)^{2 N} \operatorname{l\eta }\right]^{1 / 2}
$$

In the normalized functions, (21) and (22), the strain gradient length parameter $l$ has been taken for normalizing the other length quantities and coordinates. Usually, another length parameter, the half height of the plastic zone for conventional material in small scale yielding, $R_{\mathrm{P}}$, is used for normalization,

$$
R_{\mathrm{P}}=\frac{K_{\mathrm{I}}^{2}}{3 \pi \sigma_{\mathrm{Y}}^{2}}
$$

such that the normalized normal traction and the effective stress can be expressed as

$$
\begin{aligned}
& \frac{t_{2}}{\sigma_{\mathrm{Y}}}=f^{\prime}\left(\frac{x_{1}}{R_{\mathrm{P}}} ; \frac{E}{\sigma_{\mathrm{Y}}}, v, N, \frac{l_{\varepsilon}}{l}, \frac{l}{R_{\mathrm{P}}}\right) \\
& \frac{\sigma_{\mathrm{e}}}{\sigma_{\mathrm{Y}}}=g^{\prime}\left(\frac{x_{1}}{R_{\mathrm{P}}} ; \frac{E}{\sigma_{\mathrm{Y}}}, v, N, \frac{l_{\varepsilon}}{l}, \frac{l}{R_{\mathrm{P}}}\right)
\end{aligned}
$$

\subsection{Crack tip field solutions}

In the solution in the forms of (21) and (22), or (25) and (26), there are too many independent and nondimensional quantities included. For simplicity, in most cases of the present analyses, take the material parameters, $E / \sigma_{\mathrm{Y}}=500, v=0.3$ and $N=0.2$ fixed, and discuss the influences of the other parameters. In 

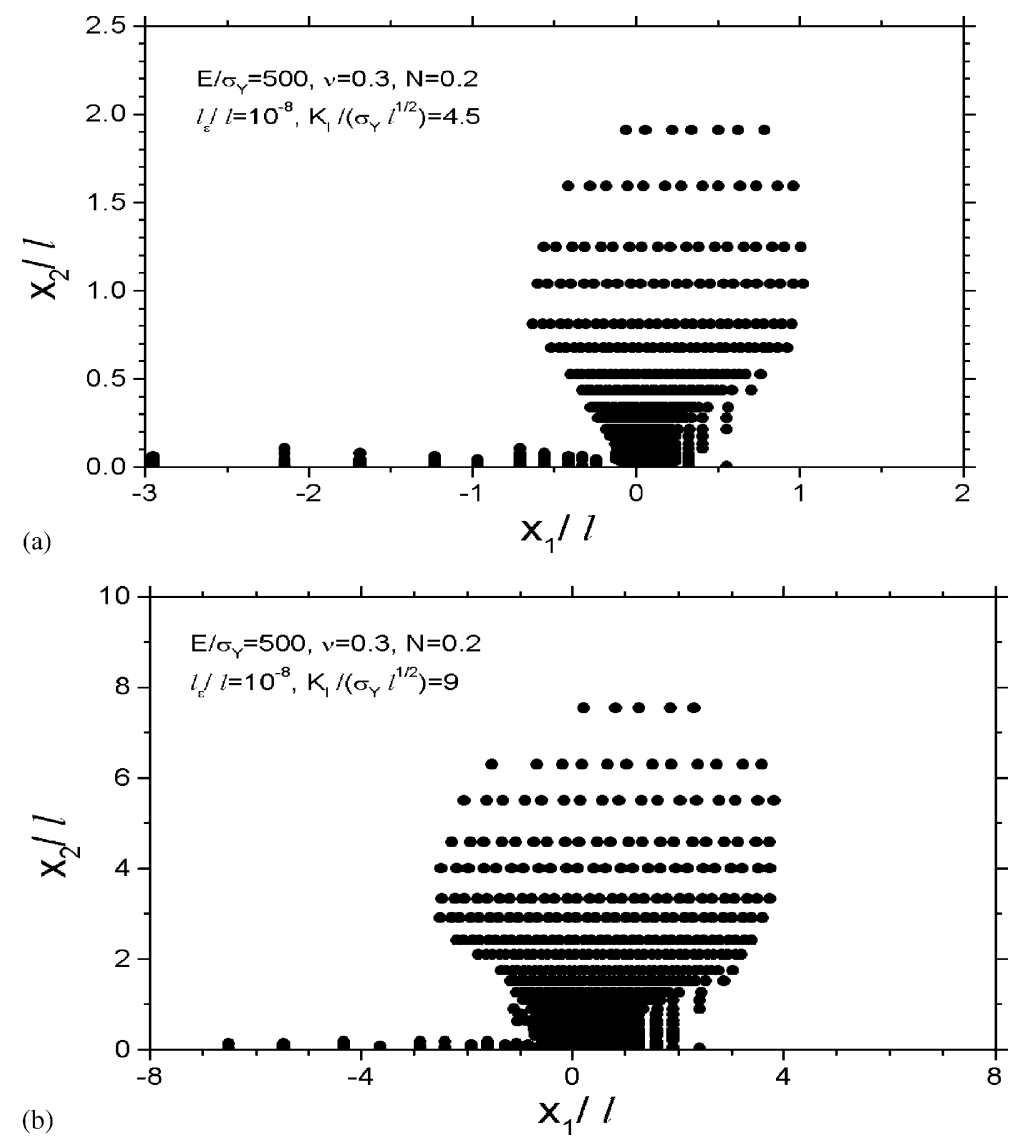

Fig. 3. Active plastic zones for two load levels.

order to check whether the strain gradient effect occurs within a small area surrounded by the conventional plastic zone and to check whether the plastic zone size is not influenced too much by the strain gradient effect, Fig. 3(a) and (b) show the active plastic zones for two normalized loading levels, $K_{\mathrm{I}} /\left(\sigma_{\mathrm{Y}} l^{1 / 2}\right)=4.5$ and 9. Considering that the plastic zone is symmetric about $x_{1}$ axis for the mode I problem, only a half zone is shown in each figure by plotting Gauss points where plastic deformation has occurred. From Fig. 3(a) and (b), the shapes of active plastic zones are very similar to each other, and to the sketch in Fig. 1(b) as well. The active plastic zones near the crack tip and around the crack surface behind the tip are displayed. As to the plastic zone size, from Fig. 3(a) and (b), the size of the plastic zone for loading level $K_{\mathrm{I}} /\left(\sigma_{\mathrm{Y}} l^{1 / 2}\right)=9$ is obviously four times that for loading level $K_{\mathrm{I}} /\left(\sigma_{\mathrm{Y}} l^{1 / 2}\right)=4.5$. This result implies that in small scale yielding, the conventional plastic zone size relation (formula (24)) is still valid for the strain gradient solid. The active plastic zone size increases in proportion to the quantity $K_{\mathrm{I}}^{2}$.

Fig. 4 shows the influence of the cell size parameter in the MSG theory on the crack tip fields. Two sets of results are shown in the Fig. 4, respectively, with solid lines and dashed lines. Two vastly different cell sizes, $l_{\varepsilon} / l=10^{-8}$ and $10^{-2}$, have led to only a small difference of the corresponding results. Therefore, one can take the ratio $l_{\varepsilon} / l$ as zero for simplicity $[37,44]$. However, for the present steady-state problem solved by adopting the iteration scheme as formulated in Eq. (19), one can not take the ratio exactly equal to zero from (A.7) and (A.8) of Appendix A, since otherwise Eq. (19) becomes singular. Subsequently, most results obtained will be based on the parameter value of $l_{\varepsilon} / l=10^{-8}$. The result based on this parameter ratio only 


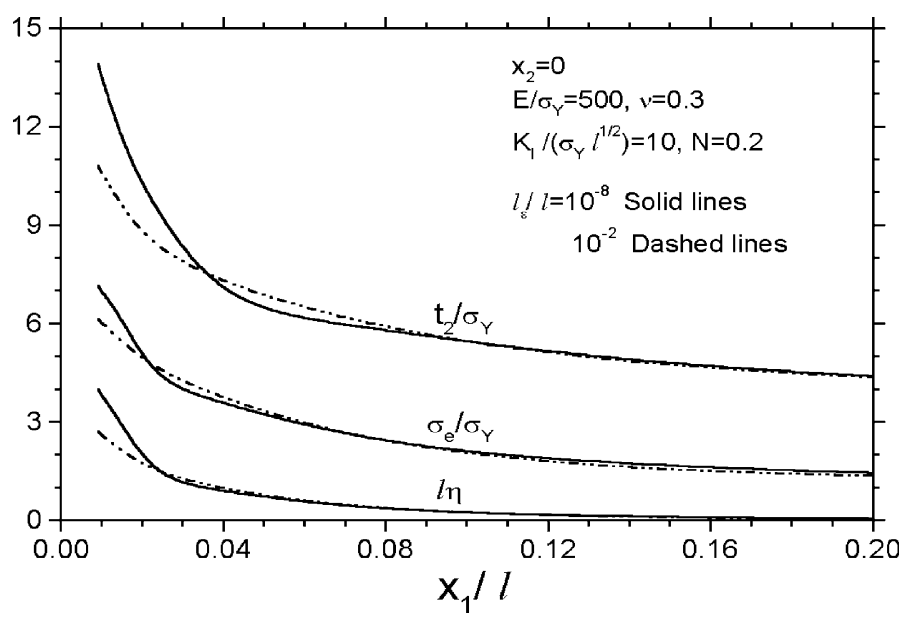

Fig. 4. The influence of cell size on the results. The results show that the cell size influence is very weak.

produces a negligible difference to the result based on a realistic value of the ratio, $l_{\varepsilon} / l$, from $10^{-3}$ to $10^{-2}$ $[33,34]$. Additionally, the behaviors of the present steady-state crack growth problem can compare with those of the stationary crack problem predicted by Qiu et al. [44].

Fig. 5(a) and (b) show the distribution of the normalized separation traction $t_{2} / \sigma_{\mathrm{Y}}$ and effective stress $\sigma_{\mathrm{e}} / \sigma_{\mathrm{Y}}$ along the plane $x_{2}=0$ ahead of crack tip for several load levels. From Fig. 5, it is clear that the distributions of $t_{2} / \sigma_{\mathrm{Y}}$ and $\sigma_{\mathrm{e}} / \sigma_{\mathrm{Y}}$ undergo a large increase for $x_{1}<0.06 l$. However, for $x_{1}>0.1 l$, both distributions $t_{2} / \sigma_{\mathrm{Y}}$ and $\sigma_{\mathrm{e}} / \sigma_{\mathrm{Y}}$ change very slowly with increasing normalized coordinate $x_{1} / l$. Reader may probably present a question here: what is the strain gradient sensitive region? However, since the strain gradient length parameter $l$ is taken as the normalizing quantity for coordinate in Fig. 5, it is difficult to discuss the size of the strain gradient sensitive zone. This question will be answered later in the discussion of Fig. 8 when $R_{\mathrm{P}}$ is taken as the normalizing quantity for coordinate. The crack tip fields for different values of $N$ are shown in Fig. 6(a)-(c). From Fig. 6(a) and (b), the strength of the crack tip stress field is sensitive to the value of material hardening exponent. For a weakly hardening material, the strength of crack tip stress field is quite low. From Fig. 6(c), the effective plastic strain has a quick increase for $x_{1}<0.05 l$. For comparison with conventional elastic-plastic solution given by Varias and Shih [18], a result considering the strain gradient effect for $N=0.1$ and $E / \sigma_{\mathrm{Y}}=300$ is added in Fig. 6(c). Varias and Shih [18] calculated the effective plastic strain distributed along the plane ahead of the crack tip for $x_{1} / l \geqslant 0.2\left(l=K_{\mathrm{I}}^{2} / 100 \sigma_{\mathrm{Y}}^{2}\right.$ as given in Fig. 6) using the conventional theory. Their result gives $E \varepsilon^{\mathrm{p}} / \sigma_{\mathrm{Y}}=3.390$ at $x_{1} / l=0.2$ from Fig. 5(a) in Varias and Shih [18]. However, the corresponding value predicted using the present theory from Fig. 6(c) is 1.252 . Therefore, more blunting of crack tip shape is predicted from the conventional theory than that from the strain gradient theory.

In order to investigate clearly the effect of length parameter $l$ in the MSG strain gradient theory, another length parameter, $R_{\mathrm{P}}$, the half height of the plastic zone in small scale yielding, is taken as the normalizing length quantity. Fig. 7 shows surface opening profile behind and near the tip during crack steady-state growth. From Fig. 7 , considering strain gradient effect $\left(l / R_{\mathrm{P}}=2\right)$, crack tip becomes considerably sharp, even for a weakly hardening material $(N=0.001)$. With increasing $l$, crack tip failure behavior tends to be a cleavage separation. In Fig. $7, l / R_{\mathrm{P}}=0.0$ corresponds to the results of the conventional elastic-plastic theory, which imply strong crack tip blunting. Fig. 8 shows the separation traction distributions along the plane $x_{2}=0$ ahead of the crack tip with the coordinate $x_{1} / R_{\mathrm{P}}$ for several values of $l / R_{\mathrm{P}}$. For comparison, the result corresponding to the conventional elastic-plastic $J_{2}$-flow theory, or for $l / R_{\mathrm{P}}=0$, is also shown in 

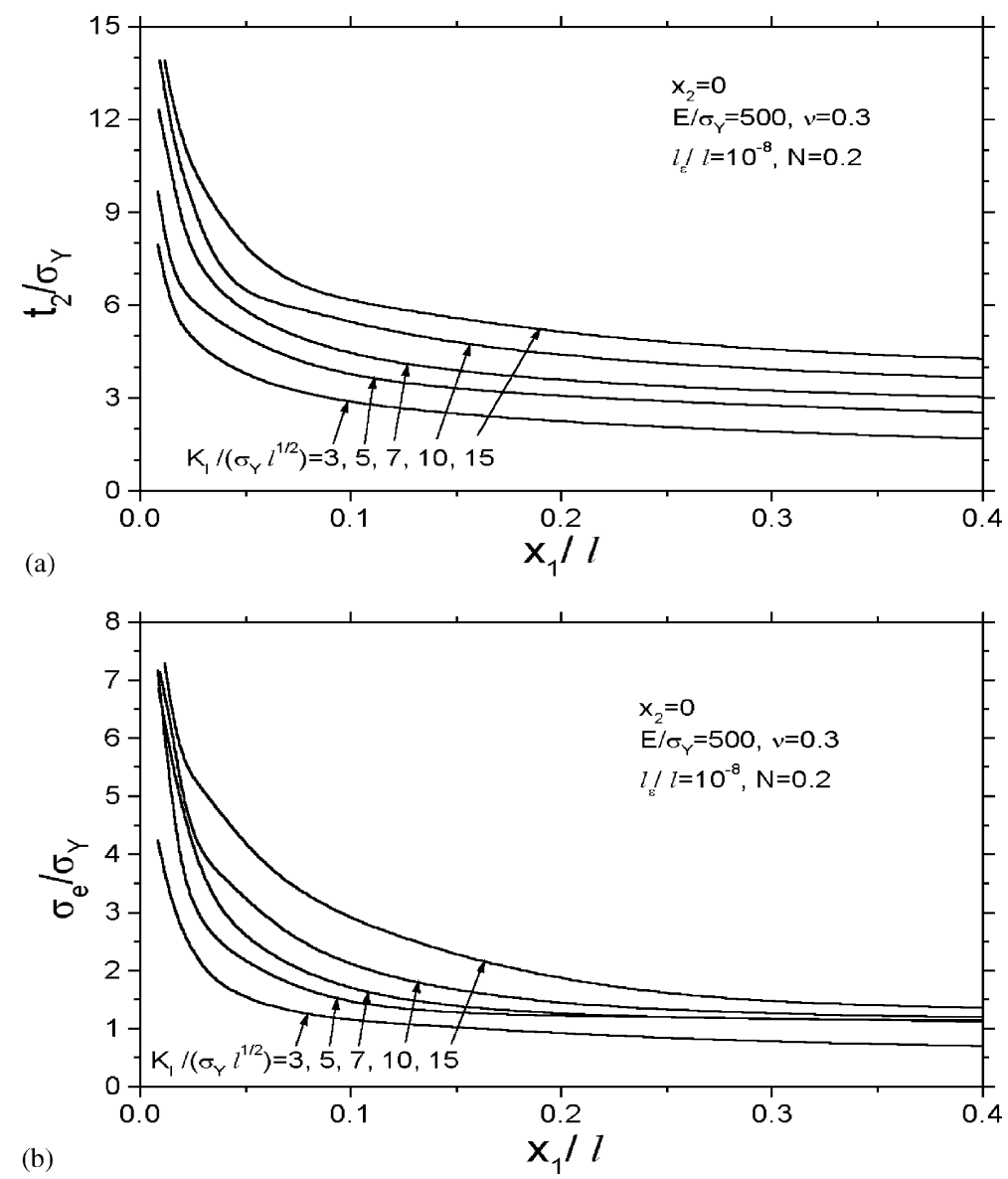

Fig. 5. The crack tip fields for different load levels based on the MSG theory. (a) Distributions of separation tractions, and (b) distributions of effective stress.

Fig. 8. For comparing the present results with the corresponding results based on the SG strain gradient flow theory [30,39], in Fig. 8 the material parameter value $E / \sigma_{\mathrm{Y}}=300$ is taken into account. The SG theory results from Wei and Hutchinson [39] are also shown in Fig. 8. From Fig. 8, the normal traction on the plane $x_{2}=0$ ahead of the crack tip achieves a bigger value near the crack tip with increasing $l$, correspondingly, with increasing length parameter $l / R_{\mathrm{P}}$. For example, at $x_{1}=0.01 R_{\mathrm{P}}$, the normal traction achieves a value as high as 12 times the yield stress for $l / R_{\mathrm{P}}=4$. However, the corresponding value at the same point $\left(x_{1}=0.01 R_{\mathrm{P}}\right)$ predicted from the conventional elastic-plastic theory $\left(l / R_{\mathrm{P}}=0.0\right)$ is only 6 times the yield stress. Now, let us discuss the strain gradient sensitive region. From the results of the MSG strain gradient theory shown in Fig. 8, the strain gradient theory results $\left(l / R_{\mathrm{P}}=0.3-4\right)$ deviate from the conventional theory result $\left(l / R_{\mathrm{P}}=0\right)$ considerably for $x_{1} / R_{\mathrm{P}}<0.04$, so that this region can be taken as the strain gradient sensitive zone.

It is interesting to compare the results using the MSG theory with the corresponding results obtained by Wei and Hutchinson [39] using the Fleck-Hutchinson SG strain gradient plasticity flow theory in Fig. 8. Obviously, the length parameter $l$ in the MSG theory must be considerably larger than that in the FleckHutchinson SG theory in order for results predicted by both theories to be matched with each other. Fig. 9 

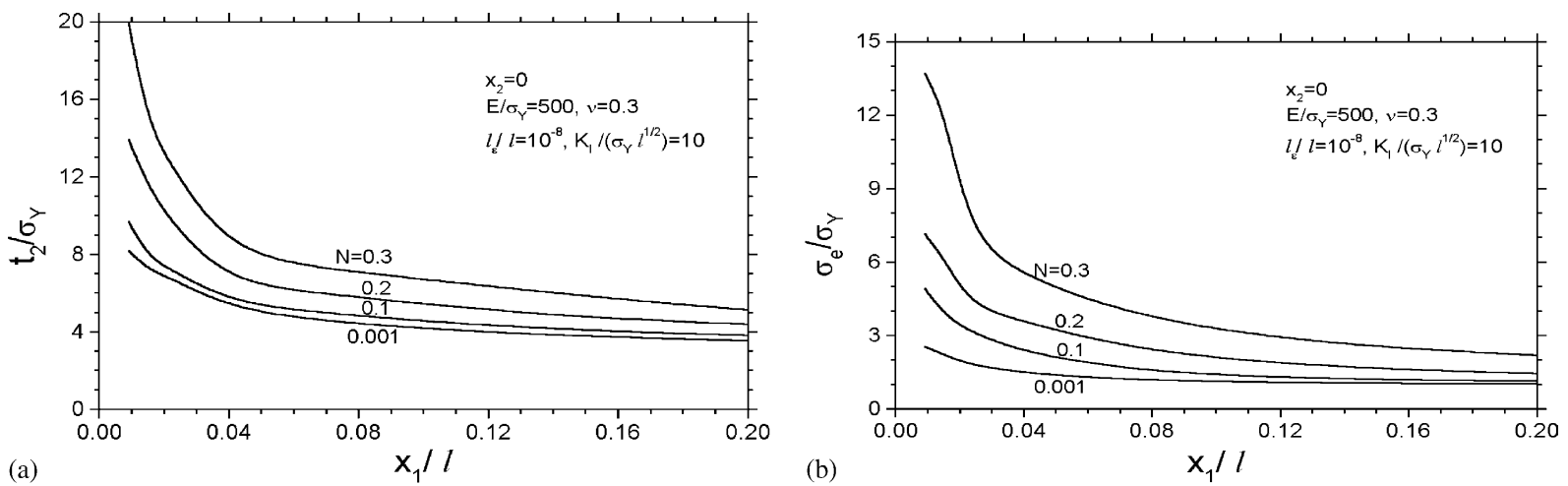

(a)

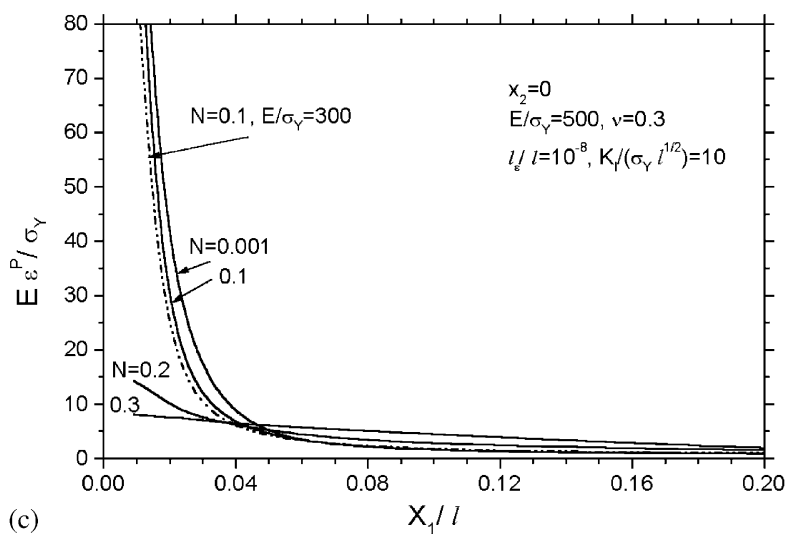

Fig. 6. The crack tip fields for different plastic hardening exponents based on the MSG theory. (a) Distributions of separation tractions, (b) distributions of effective stress and (c) distributions of effective plastic strain.

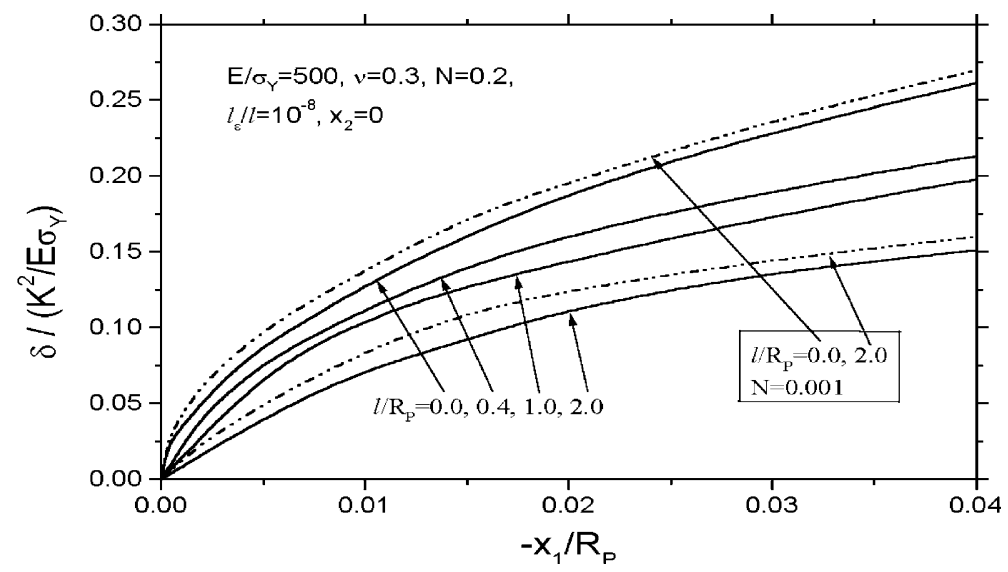

Fig. 7. The crack surface opening profile near the crack tip for different length parameters and for different hardening exponents.

shows the cell size effect for two sets of ratio $l_{\varepsilon} / l$ when the plastic zone size is taken as the normalizing length quantity. The cell size effect is quite small, as is expected. 


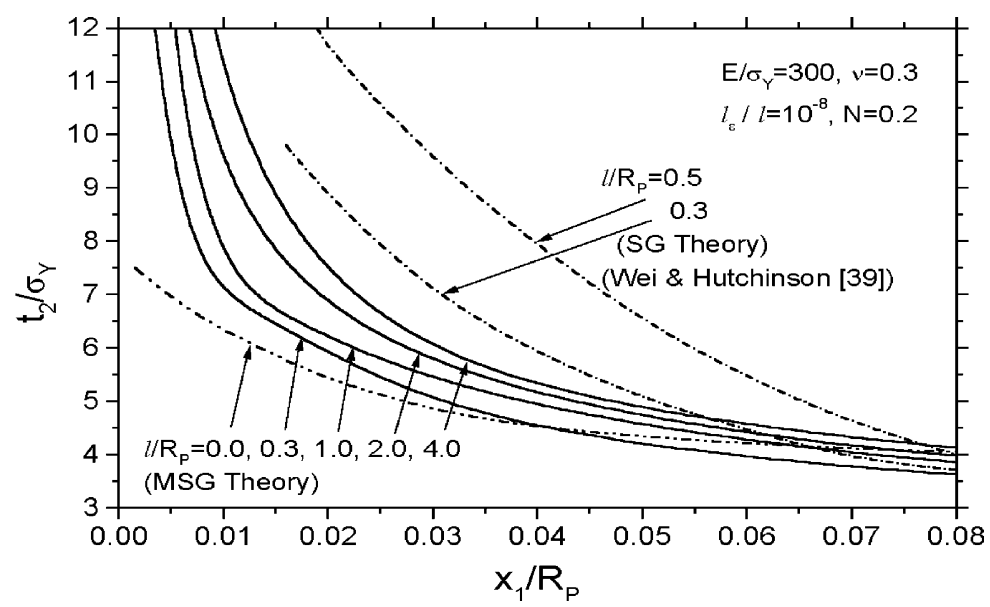

Fig. 8. The distributions of separation traction near the tip for several length parameter values. The half height of active plastic zone is taken as the normalizing length quantity. Both theory results are compared.

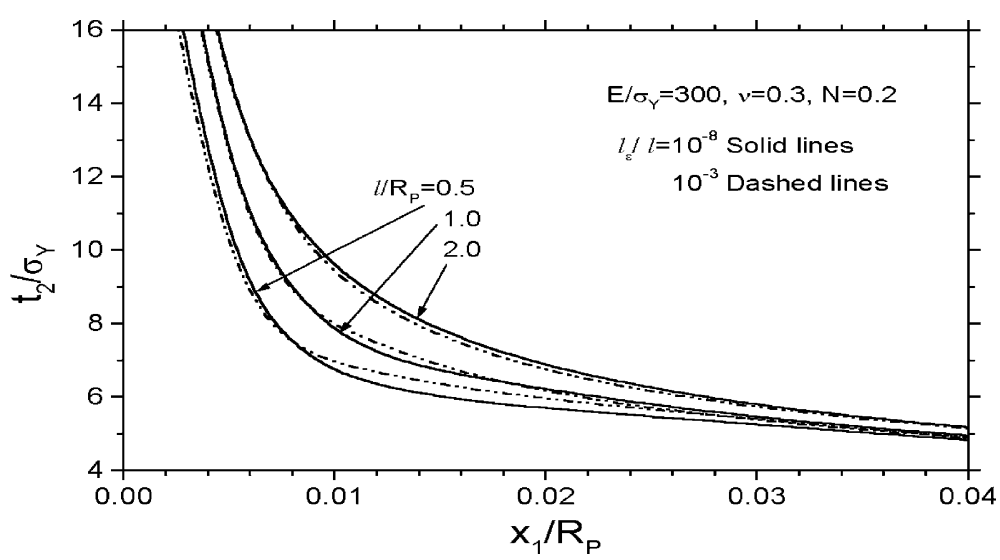

Fig. 9. The influence of cell size on the separation traction near the crack tip is shown and the influence is weak.

\section{Steady-state fracture toughness}

For the fracture process zone models, earlier work [47] gave a formulation in terms of the process zone at the crack tip for elastic crack growth problem. A great progress was made since the fracture process zone (or called EPZ) model was successfully applied to the elastic-plastic fracture process description [2,3,48]. In this section, the MSG solid is taken as the description of the elastic-plastic solid in the EPZ model. The traction-separation law characterizing the fracture process is prescribed as a boundary condition along the plane ahead of the crack tip, as depicted in Fig. 10. The continuum description of the elastic-plastic solid holds everywhere outside the extended fracture plane. Heretofore, conventional $J_{2}$ flow theory has been used to describe the solid when strain gradient effect is negligible. Attention here will be focused on steadystate, mode I plane strain toughness under small scale yielding conditions. Based on the discussion in the previous sections and the results of the last section, the expectation is that the strain gradient effect will elevate the stress level within the fracture process zone. 

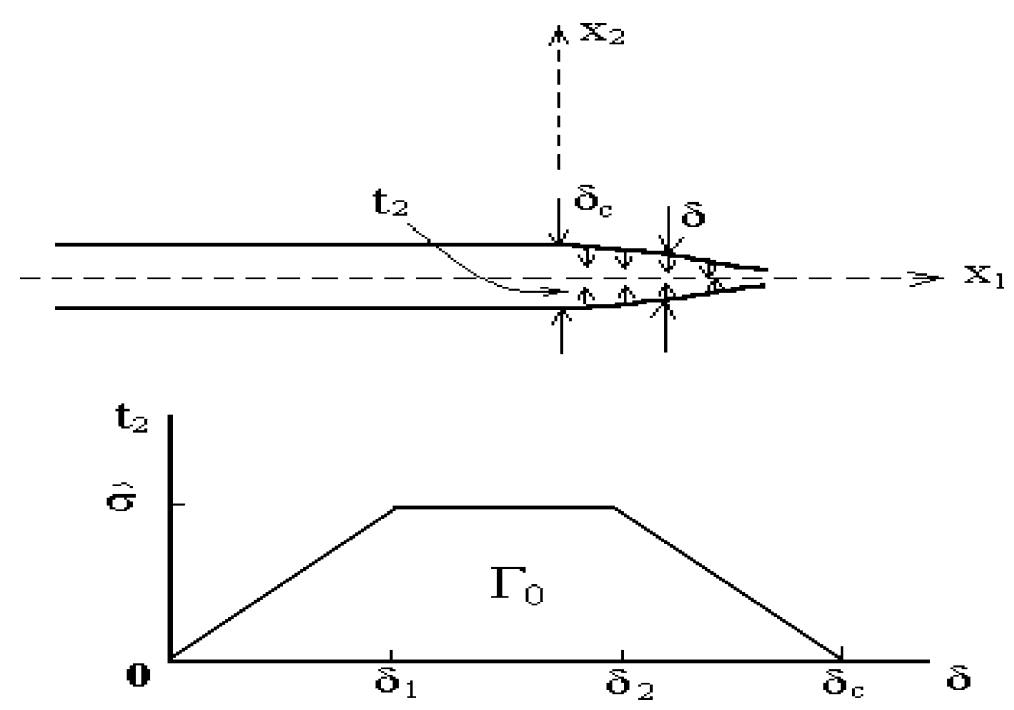

Fig. 10. EPZ model for determination of steady-state toughness. The traction-separation relation characterizing the fracture process is applied along the plane ahead of the tip and is specified by several model parameters.

The form of the traction-separation law shown in Fig. 10 is exactly the same as that employed in Tvergaard and Hutchinson [2]. However, within the context of the MSG theory, it is $t_{2}$, not $\sigma_{22}$, that is work conjugate to the crack opening separation $\delta=u_{2}\left(x_{1}, 0^{+}\right)-u_{2}\left(x_{1}, 0^{-}\right)$. Thus, in the present version of the EPZ model, the relation between $t_{2}$ and $\delta$ in Fig. 10 is prescribed as the condition along the plane ahead of the crack tip (i.e. on $x_{2}=0$ for $x_{1}>0$ ). The work of fracture per unit area, $\Gamma_{0}$, is related to $\hat{\sigma}$ and $\delta$ by

$$
\Gamma_{0}=\int_{0}^{\delta_{\mathrm{c}}} t_{2} \mathrm{~d} \delta=\frac{1}{2} \hat{\sigma} \delta_{\mathrm{c}}\left(1+\lambda_{2}-\lambda_{1}\right)
$$

where $\lambda_{1}=\delta_{1} / \delta_{\mathrm{c}}$ and $\lambda_{2}=\delta_{2} / \delta_{\mathrm{c}}$ characterize the shape of traction-separation relation in the EPZ model.

The numerical method discussed in Section 3 is applied to the previous steady-state problem, combined with additional condition from the EPZ model. Now, the iteration scheme must satisfy the traction-separation relation ahead of the crack tip and the level of the remote stress intensity $K_{\mathrm{I}}$ must be adjusted such that the propagation condition at the tip is met, i.e.

$$
\delta=\delta_{\mathrm{c}} \quad \text { at } \quad x_{1}=0
$$

The aim here is to obtain the relation between $K_{\mathrm{ss}}$ and the parameters specifying the fracture process and the MSG solid. The results will be presented using the equivalent energetic measure of steady-state toughness (Fig. 1)

$$
\Gamma_{\mathrm{ss}}=\frac{1-v^{2}}{E} K_{\mathrm{ss}}^{2}
$$

Accordingly, this quantity measures the total fracture work and $\Gamma^{\mathrm{p}}=\Gamma_{\mathrm{ss}}-\Gamma_{0}$ is the plasticity contribution to the fracture work.

About the EPZ model, the shape parameters, $\lambda_{1}$ and $\lambda_{2}$, have been shown to be relatively unimportant $[2,3]$. Thus, the EPZ model provides two independent dominating parameters $\left(\Gamma_{0}, \hat{\sigma}\right)$ for crack growth and another parameter, crack tip opening displacement $\delta_{\mathrm{c}}$, related with them through (27). $\Gamma_{0}$ characterizes the crack separation work per unit length of the crack tip advance. $\hat{\sigma}$ is the maximum separation strength near 
the tip during crack growth. In the present research, assume that the two-parameter $\left(\Gamma_{0}, \hat{\sigma}\right)$ criterion characterized by the EPZ model is still valid for crack growth in the strain gradient solid. Considering that there will be many parameters in the analysis, we take $\left(\lambda_{1}, \lambda_{2}\right)=(0.15,0.5)$ for simplicity in the present research.

Dimensional considerations now give a parametrical relation about the normalized steady-state fracture toughness as

$$
\frac{\Gamma_{\mathrm{ss}}}{\Gamma_{0}}=F\left(\frac{E}{\sigma_{\mathrm{Y}}}, v, N, \frac{\hat{\sigma}}{\sigma_{\mathrm{Y}}}, \frac{l_{\varepsilon}}{l}, \frac{l}{R_{0}}\right)
$$

where a length parameter $R_{0}$ is introduced, and its definition is

$$
R_{0}=\frac{E \Gamma_{0}}{3 \pi\left(1-v^{2}\right) \sigma_{\mathrm{Y}}^{2}}
$$

Besides $l$ and $l_{\varepsilon}, R_{0}$ is the only length quantity in the model. It can be interpreted as an estimate of the half height of the plastic zone in the limit that $\Gamma_{\mathrm{ss}}$ is only slightly greater than $\Gamma_{0}$. Equivalently, it can be thought of as the estimate of the half height of the plastic zone when $K_{\mathrm{I}}=\sqrt{E \Gamma_{0} /\left(1-v^{2}\right)}$ is applied at the remote boundary. Note that $R_{\mathrm{P}}$ defined by (24) is precisely equal to $\left(\Gamma_{\mathrm{ss}} / \Gamma_{0}\right) R_{0}$. From (27), crack tip opening displacement $\delta_{\mathrm{c}}$ closely depends on the process zone dominating parameters $\left(\Gamma_{0}, \hat{\sigma}\right)$. Using $R_{0}$ definition in Eq. (31), one obtains simply

$$
\frac{\delta_{\mathrm{c}}}{R_{0}}=\frac{6 \pi\left(1-v^{2}\right)}{\left(1+\lambda_{2}-\lambda_{1}\right)\left(E / \sigma_{\mathrm{Y}}\right)\left(\hat{\sigma} / \sigma_{\mathrm{Y}}\right)}
$$

and roughly estimates $\delta_{\mathrm{c}} \approx(0.01-0.05) R_{0}$ for the conventional metal materials and for separation strength $\hat{\sigma} \approx(1-5) \sigma_{\mathrm{Y}}$. Therefore, the length parameters in the EPZ model such as $\delta_{1}=\lambda_{1} \delta_{\mathrm{c}}$ and $\delta_{2}=\lambda_{2} \delta_{\mathrm{c}}$ are two orders smaller than the plastic zone size of the small scale yielding case, $R_{0}$. This is likely to be a reason why the values of $\delta_{1}$ and $\delta_{2}$ play an insignificant role in the EPZ model. The length parameter $l$ in the MSG strain gradient plasticity is of the same order as $R_{0}$ (see subsequent analysis result). Thus, the value of $\delta_{\mathrm{c}}$ is also two orders smaller than material length parameter. Therefore, we expect that the two-parameter $\left(\Gamma_{0}, \hat{\sigma}\right)$ criterion characterized by the EPZ model is still valid for crack growth in the strain gradient solid.

The role of strain gradient hardening in determining toughness is seen in Fig. 11 for the case of a solid with moderately high strain hardening, $N=0.2$. From Fig. 11, the increase of the steady-state toughness normalized with the separation work, with the maximum separation strength, is very abrupt for $\hat{\sigma} / \sigma_{\mathrm{Y}}>2$. In Fig. 11, the curves corresponding to several normalized length parameters are plotted. The conventional elastic-plastic $J_{2}$-flow theory result, corresponding to $l / R_{0}=0$, implies that under steady-state crack growth, the separation strength near the crack tip never obtains a high value (around five times the yield stress). If a material or a bi-material interface bonded by two materials has a higher separation strength than five times the yield stress, from the conventional theory result, the steady-state crack growth process becomes difficult to be realized, or even impossible. However, using the strain gradient plasticity theory or considering the strain gradient effects, the predicted results display that the separation traction ahead of the crack tip is elevated very much, thereby allowing higher peak separation stresses to be overcome. It is also interesting to compare the results based on the MSG theory to those based on the Fleck-Hutchinson SG strain gradient plasticity theory [39], also shown in Fig. 11. Firstly, the trends of curves $\Gamma_{\mathrm{ss}} / \Gamma_{0} \sim \hat{\sigma} / \sigma_{\mathrm{Y}}$ for both theories are very similar; secondly, the length scale parameters in both theories seems to have an approximate relation

$$
l_{\mathrm{MSG}} \approx(4-5) l_{\mathrm{SG}}
$$




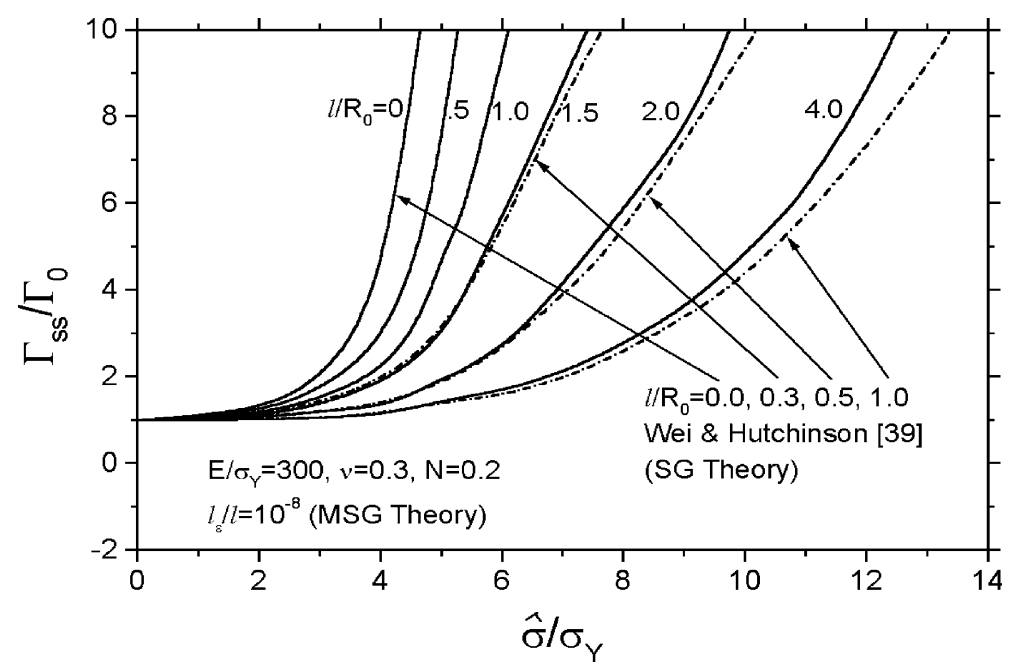

Fig. 11. Steady-state fracture toughness changes with the separation strength and the normalized length parameter based on two strain gradient theories: The MSG theory and the SG theory of Fleck-Hucthinson's strain gradient theory. Through comparison a relation of length parameters in the two theories is set up.

where $l_{\mathrm{MSG}}$ is the length parameter of the MSG theory [33,34], and $l_{\mathrm{SG}}$ is the length parameter of the SG flow theory [30,39]. Under this relation, both theory results of the toughness-strength curves (Fig. 11) merge approximately. From previous studies for both theories by Gao et al. [33], Huang et al. [34], Begley and Hutchinson [36], Wei and Hutchinson [9], Wei [42] and Wei et al. [26], the approximate relation between length parameters (formula (33)) is reasonable.

\section{Concluding remarks}

Mode I steady-state crack growth has been analyzed under plane strain conditions in small scale yielding. The elastic-plastic solid is characterized by the mechanism-based strain gradient plasticity theory. The following conclusions have been obtained:

1. For the steady-state crack growth, the crack tip separation stress achieves considerably high value within a sensitive zone of the strain gradient.

2. The steady-state fracture toughness is very sensitive to the material length parameter in the strain gradient plasticity theory and the separation strength of the EPZ model.

3. The length parameter in the MSG theory is about 4-5 times the corresponding quantity in the FleckHutchinson strain gradient plasticity theory.

For the fracture work calculation using the EPZ model, with values of $l / R_{0}$ larger than about 2 (MSG theory), the peak separation traction well above $10 \sigma_{\mathrm{Y}}$ can be attained. Atomic separation of a metal lattice or a metal/ceramic interface typically requires a work of separation, $\Gamma_{0}$, on the order of several $\mathrm{J} \mathrm{m}^{-2}$. Using representative values for $E$ and $\sigma_{\mathrm{Y}}$ for the typical metals, one finds values of $R_{0}$ in the range from about 0.1 to $1 \mu \mathrm{m}$. The available experimental data indicate that $l$ in Fleck and Hutchinson theory [30] is likely to be on the order of half or $1 \mu \mathrm{m}[20,26,36]$, the corresponding length parameter value in the MSG theory is likely on the order of several micro-meters from the discussion in the last section. Therefore, the values of $l$ 
in the MSG theory at least as large as several times $R_{0}$ must be expected for fracture processes based on separation at the atomic scale.

\section{Acknowledgements}

The work is supported by National Science Foundations of China through Grants 19925211; jointly supported by Chinese Academy of Sciences through "Bai Ren" Project and Chinese Ministry of Education.

\section{Appendix A. The compact form of the MSG flow theory}

The MSG flow theory can be expressed with a compact form

$$
\left\{\begin{array}{l}
\dot{\sigma}_{i j}=A_{i j k l} \dot{\varepsilon}_{k l}+B_{i j k l m} \dot{\eta}_{k l m} \\
\dot{\tau}_{i j k}=A_{i j k l m}^{\prime} \dot{\varepsilon}_{l m}+B_{i j k l m n}^{\prime} \dot{\eta}_{l m n}
\end{array}\right.
$$

and the expressions of coefficient tensors can be obtained from (6) as

$$
\begin{aligned}
A_{i j k l}= & L_{i j k l}-\frac{3 \alpha^{\prime} \mu \sigma_{i j}^{\prime} \sigma_{k l}^{\prime}}{\sigma_{\mathrm{e}}^{2}(1+a)} \\
B_{i j k l m}= & \frac{\alpha^{\prime} l \sigma_{0}^{2} \sigma_{i j}^{\prime} \eta_{k l m}^{\prime}}{8 \eta \sigma_{\mathrm{e}}^{2}(1+a)} \\
A_{i j k l m}^{\prime}= & \frac{3 \alpha^{\prime} \mu}{2 \sigma_{\mathrm{e}}^{2}(1+a)} G_{i j k p q}\left[\frac{3(2+a) / 2+e \sigma_{\mathrm{e}} /(2 \mu)}{\sigma_{\mathrm{e}}^{2}(1+a)} \sigma_{p q}^{\prime} \sigma_{l m}^{\prime}-\left(\delta_{p l} \delta_{q m}-\frac{1}{3} \delta_{p q} \delta_{l m}\right)\right] \\
& -\frac{3 \alpha^{\prime} \mu}{\sigma_{\mathrm{e}}^{2}(1+a)}\left(\tau_{i j k}-\frac{K l_{\varepsilon}^{2}}{6} \eta_{i j k}^{H}\right) \sigma_{l m}^{\prime} \\
B_{i j k l m n}^{\prime}= & \Pi_{i j k l m n}-\alpha^{\prime}\left\{\frac{\mu l_{\varepsilon}^{2}}{16 \sigma_{\mathrm{e}}^{2}(1+a)}\left[\left(\sigma_{k i}^{\prime} \delta_{j l}+\sigma_{k j}^{\prime} \delta_{i l}\right) \sigma_{m n}^{\prime}+\left(\sigma_{k i}^{\prime} \delta_{j m}+\sigma_{k j}^{\prime} \delta_{i m}\right) \sigma_{l n}^{\prime}\right]\right. \\
& \left.+\frac{\left(6+3 a+e \sigma_{\mathrm{e}} / \mu\right) l \sigma_{0}^{2}}{32 \eta \sigma_{\mathrm{e}}^{4}(1+a)^{2}} G_{i j k p q} \sigma_{p q}^{\prime} \eta_{l m n}^{\prime}-\frac{l \sigma_{0}^{2}}{8 \eta \sigma_{\mathrm{e}}^{2}(1+a)}\left(\tau_{i j k}-\frac{K l_{\varepsilon}^{2}}{6} \eta_{i j k}^{H}\right) \eta_{l m n}^{\prime}\right\}
\end{aligned}
$$

where

$$
\begin{aligned}
& L_{i j k l}=\frac{E}{2(1+v)}\left[\delta_{i j} \delta_{k l}+\delta_{i l} \delta_{j k}+\frac{2 v}{(1-2 v)} \delta_{i j} \delta_{k l}\right] \\
& \Pi_{i j k l m n}=\frac{\mu l_{\varepsilon}^{2}}{12}\left\{\delta_{i l} \delta_{j m} \delta_{k n}+\frac{1}{2}\left(\delta_{i m} \delta_{j n}+\delta_{j m} \delta_{i n}\right) \delta_{k l}+\frac{1}{8}\left(\frac{2 K}{\mu}-\frac{4}{3}\right)\left[\left(\delta_{i k} \delta_{j l}+\delta_{j k} \delta_{i l}\right) \delta_{m n}+\left(\delta_{i k} \delta_{j m}+\delta_{j k} \delta_{i m}\right) \delta_{l n}\right]\right\}
\end{aligned}
$$

Other parameters and variables have been defined in formulas from (6) to (12).

For the elastic case, the coefficient tensors become

$$
A_{i j k l}=L_{i j k l}, \quad B_{i j k l m}=0, \quad A_{i j k l m}^{\prime}=0, \quad B_{i j k l m n}^{\prime}=\Pi_{i j k l m n}
$$




\section{References}

[1] Hutchinson JW, Suo Z. Mixed mode cracking in layered materials. In: Hutchinson JW, Wu TY, editors. Advances in applied mechanics, vol. 29. New York: Academic; 1992. p. 63-191.

[2] Tvergaard V, Hutchinson JW. The relation between crack growth resistance and fracture process parameters in elastic-plastic solids. J Mech Phys Solids 1992;40:1377-97.

[3] Tvergaard V, Hutchinson JW. The influence of plasticity on mixed mode interface toughness. J Mech Phys Solids 1993;41:111935.

[4] Bagchi A, Lucas GE, Suo Z, Evans AG. A new procedure for measuring the decohesion energy of thin ductile films on substrates. J Mater Res 1994;9:1734-41.

[5] Bagchi A, Evans AG. The mechanics and physics of thin film decohesion and its measurement. Interf Sci 1996;3:169-93.

[6] Beltz GE, Rice JR, Shih CF, Xia L. A self-consistent model for cleavage in the presence of plastic flow. Acta Mater 1996;44: 3943-54.

[7] Wei Y, Hutchinson JW. Nonlinear delamination mechanics for thin films. J Mech Phys Solids 1997;45:1137-59.

[8] Wei Y, Hutchinson JW. Interface strength, work of adhesion and plasticity in the peel test. Int J Fract 1998;93:315-33.

[9] Wei Y, Hutchinson JW. Models of interface separation accompanied by plastic dissipation at multiple scales. Int J Fract 1999;95:1-17.

[10] Lipkin DM, Clarke DR, Evans AG. Effect of interfacial carbon on adhesion and toughness of gold-sapphire interface. Acta Mater 1998;46:4835-50.

[11] Reimanis IE, Dalgleish BJ, Evans AG. The fracture resistance of a model metal/ceramic interface. Acta Metall Mater 1991;39:3133-41.

[12] Lane M, Dauskardt RH, Vainchtein A, Gao H. Plasticity contributions to interface adhesion in thin-film interconnect structures. J Mater Res 2000;15:2758-69.

[13] Dean RH, Hutchinson JW. Quasi-static steady crack growth in small scale yielding. In: Fracture mechanics. ASTM STP 700. 1980. p. 383-405.

[14] Parks DM, Lam PS, McMeeking RM. Some effects of inelastic constitutive models on crack tip fields in steady quasistatic growth. In: Francois D, editor. Proc of 5th Int Conf on Fracture. Advances in fracture research, 5. Amsterdam: Pergamon; 1981. p. 260714.

[15] Gao YC, Hwang KC. Elastic-plastic fields in steady crack growth. In: Three dimensional constitutive relations and ductile fracture, IUTAM Symposium, Dourdan, France, June 2-5, 1980. North-Holland.

[16] Drugan WJ, Rice JR, Sham TL. Asymptotic analysis of growing plane strain tensile cracks in elastic-ideally plastic solids. J Mech Phys Solids 1982;30:447-73.

[17] Hwang KC, Luo XF. Near-tip fields of growing cracks and resistance curves. Mech Mater 1989;7:271-8.

[18] Varias AG, Shih CF. Quasi-static crack advance under a range of constraints-steady-state fields based on a characteristic length. J Mech Phys Solids 1993;41:835-61.

[19] Suo Z, Shih CF, Varias AG. A theory for cleavage cracking in the presence of plastic flow. Acta Metall Mater 1993;41:151-1557.

[20] Evans AG, Hutchinson JW, Wei Y. Interface adhesion: effects of plasticity and segregation. Acta Mater 1999;47:4093-113.

[21] Raynolds JE, Smith JR, Zhao GL, Srolovitz DJ. Adhesion in NiAl-Cr from first principles. Phys Rev B 1996;53:13883-90.

[22] Hong T, Smith JR, Srolovitz DJ. Metal-ceramic adhesion-a first principle study of MgO/Al and MgO/Ag. J Adhes Sci Technol 1994;8:837-51.

[23] Stelmashenko NA, Walls MG, Brown LM, Milman YV. Microindentations on W and Mo oriented single crystals: An STM study. Acta Metall Mater 1993;41:2855-65.

[24] Ma Q, Clarke DR. Size dependent hardness of silver single crystals. J Mater Res 1995;10:853-63.

[25] McElhaney KW, Vlassak JJ, Nix WD. Determination of indenter tip geometry and indentation contact area for depth-sensing indentation experiments. J Mater Res 1998;13:1300-6.

[26] Wei Y, Wang X, Wu X, Bai Y. Theoretical and experimental researches of size effect in micro-indentation test. Sci China (Series A) 2001;44:74-82.

[27] Fleck NA, Muller GM, Ashby MF, Hutchinson JW. Strain gradient plasticity: theory and experiments. Acta Metall Mater 1994;42:475-87.

[28] Stolken JS, Evans AG. A microbend test method for measuring the plasticity length scale. Acta Mater 1998;46:5109-15.

[29] Fleck NA, Hutchinson JW. A phenomenological theory for strain gradient effects in plasticity. J Mech Phys Solids 1993;41:182557.

[30] Fleck NA, Hutchinson JW. Strain gradient plasticity. In: Hutchinson JW, Wu TY, editors. Advances in applied mechanics, vol. 33. New York: Academic; 1997. p. 295-361.

[31] Aifantis EC. On the microstructural origin of certain inelastic models. Trans ASME J Engng Mater Technol 1984;106:326-30.

[32] Chen SH, Wang TC. Strain gradient plasticity with couple stress for crystalline solids. Eur J Mech A/Solids 2001;20:739-56. 
[33] Gao H, Huang Y, Nix WD, Hutchinson JW. Mechanism-based strain gradient plasticity—I. Theory. J Mech Phys Solids 1999;47:1239-63.

[34] Huang Y, Gao H, Nix WD, Hutchinson JW. Mechanism-based strain gradient plasticity-II. Analysis. J Mech Phys Solids 2000;48:99-128.

[35] Shu JY, Fleck NA. The prediction of a size effect in micro indentation. Int J Solids Struct 1998;35:1363-83.

[36] Begley MR, Hutchinson JW. The mechanics of size-dependent indentation. J Mech Phys Solids 1998;46:2049-68.

[37] Huang Y, Xue Z, Gao H, Nix WD, Xia ZC. A study of micro-indentation hardness tests by mechanism-based strain-gradient plasticity. J Mater Res 2000;15:1786-96.

[38] Xia ZC, Hutchinson JW. Crack tip fields in strain gradient plasticity. J Mech Phys Solids 1996;44:1621-48.

[39] Wei Y, Hutchinson JW. Steady-state crack growth and work of fracture for solids characterized by strain gradient plasticity. J Mech Phys Solids 1997;45:1253-73.

[40] Huang Y, Chen JY, Guo TF, Zhang L, Hwang KC. Analytic and numerical studies on mode I and mode II fracture in elasticplastic materials with strain gradient effects. Int J Fract 1999;100:1-27.

[41] Huang Y, Gao H, Hwang KC. Strain-gradient plasticity at the micron scale. In: Ellyin F, Provan JW, editors. Progress in mechanical behavior of materials, vol. III. 1999. p. 1051-6.

[42] Wei Y. Microscale mechanics for metal thin film delamination along ceramic substrates. Sci China (Series A) 2000;43:509-16.

[43] Wei Y. Constraint effects on the elastic-plastic fracture behaviour in strain gradient solids. Fatigue Fract Engng Mater Struct 2002;25:433-44.

[44] Qiu X, Huang Y, Wei Y, Gao H, Hwang KC. The flow theory of mechanism-based strain gradient plasticity. Mech Mater 2003;35:245-58.

[45] Nix WD, Gao H. Indentation size effects in crystalline materials: a law for strain gradient plasticity. J Mech Phys Solids 1998;46:411-25.

[46] Chen JY, Wei Y, Huang Y, Hutchinson JW, Hwang KC. The crack tip fields in strain gradient plasticity: the asymptotic and numerical analyses. Engng Fract Mech 1999;64:625-48.

[47] Barenblatt GI. The mathematical theory of equilibrium cracks in brittle fracture. Adv Appl Mech 1962;7:55-129.

[48] Needleman A. A continuum model for void nucleation by inclusion debonding. J Appl Mech 1987;54:525-31. 